\title{
Employing ambipolar oligofluorene as the charge-generation layer in time-of-flight mobility measurements of organic thin films
}

\author{
Wen-Yi Hung ${ }^{\text {a) }}$ \\ Graduate Institute of Electro-Optical Engineering, National Taiwan University, Taipei, Taiwan 106 \\ and Institute of Optoelectronic Sciences, National Taiwan Ocean University, Keelung, Taiwan 202, \\ Republic of China \\ Tung-Huei Ke, Yu-Ting Lin, and Chung-Chih $\mathrm{Wu}^{\text {b) }}$ \\ Department of Electrical Engineering, Graduate Institute of Electro-Optical Engineering, and Graduate \\ Institute of Electronics Engineering, National Taiwan University, Taipei, Taiwan 106, \\ Republic of China \\ Tsung-Hsi Hung, Teng-Chih Chao, and Ken-Tsung Wong \\ Department of Chemistry, National Taiwan University, Taipei, Taiwan 106, Republic of China \\ Chih-I Wu \\ Department of Electrical Engineering and Graduate Institute of Electro-optical Engineering, \\ National Taiwan University, Taipei, Taiwan 106, Republic of China
}

(Received 22 August 2005; accepted 11 January 2006; published online 9 February 2006)

\begin{abstract}
Strong absorption of oligofluorenes at wavelengths of a few commonly used nanosecond pulsed lasers, their bipolar transport characteristics, and high mobilities for both holes and electrons make them useful as the general and effective charge-generation material for the time-of-flight mobility measurement of organic materials. In this letter, we demonstrate the use of the terfluorene as the charge-generation material for measuring hole and electron mobilities of various organic materials. Such a scheme has the advantages of simplifying the instrumentation and reducing material consumption in the measurements. (C) 2006 American Institute of Physics.
\end{abstract}

[DOI: $10.1063 / 1.2172708]$

Organic semiconductors and optoelectronics advance rapidly in recent years. ${ }^{1-6}$ In developing organic semiconductors and devices, knowledge of their carrier-transport properties (mobilities) is essential. One common method of characterizing carrier mobilities in organic materials is the time-of-flight (TOF) transient photocurrent technique. ${ }^{7}$ In the simplest TOF configuration, the material to be measured is sandwiched between two blocking contacts, of which at least one is (semi)transparent to receive pulsed illumination (usually a nanosecond pulsed laser) for producing a thin sheet of photogenerated carriers near the electrode. Depending on the polarity of the applied bias, selected carriers (either holes or electrons) are swept across the sample and a transient photocurrent is recorded. In such a configuration, for unambiguous determination of the transit time from the TOF transient, the thickness of the organic layer is usually set one order of magnitude larger than the absorption depth (the chargegeneration region). For instance, for a material with an absorption coefficient of $10^{5} \mathrm{~cm}^{-1}$ at the excitation wavelength, the absorption depth is about 1000-2000 $\AA$ and the required sample thickness would be $\sim 1-2 \mu \mathrm{m}$. However, the material of interest may not have a large enough absorption coefficient at the available excitation wavelength. One will then face the issues of an impractically large sample thickness and a huge consumption of materials. Alternatively, one may set up an excitation light source with wavelengths tunable to match the absorption of materials, which however involves

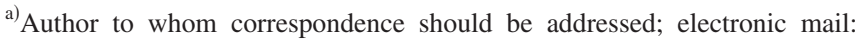
wenhung@mail.ntou.edu.tw

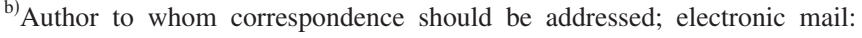
chungwu@cc.ee.ntu.edu.tw
}

more complicated and costly instrumentation.

The better alternative is to incorporate between the electrode and the material under test a thin charge-generation layer (CGL) with strong absorption coefficient at the available excitation wavelengths. ${ }^{7}$ In such a configuration as shown in Fig. 1(a), optical absorption and photocarrier generation occur in the CGL. Photogenerated carriers are injected into the material layer under test for drifting across the layer. Such a CGL-TOF technique indeed was widely used for studying carrier transport in molecularly dispersed polymers (MDPs). ${ }^{7}$ Yet, the vast amount of previous MDP work mainly dealt with unipolar charge-transport materials. Thus for the CGL's used to study MDPs, usually only the injection and transport properties for one type of carriers were considered and exact CGL's used for different materials were case dependent. Ideally, one would like to have one common CGL material that can be used for studying both holetransport and electron-transport properties of a material or different materials. As shown in Fig. 1(b), such a CGL is desired to have a strong absorption for a convenient excita-
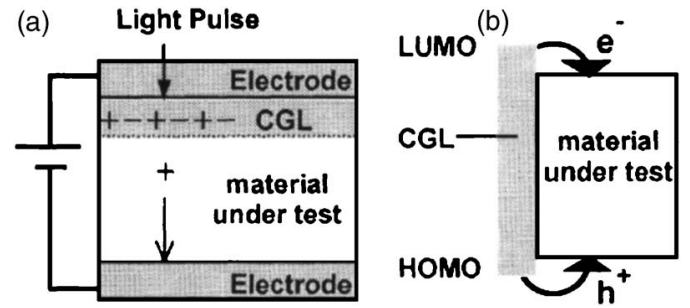

FIG. 1. (a) Schematic diagram showing the TOF sample configuration with the charge-generation layer. (b) Relative energy levels of a general chargegeneration layer and the material under test. 


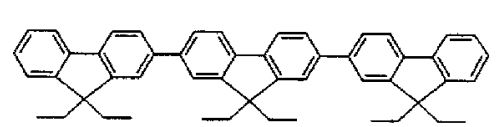

E3

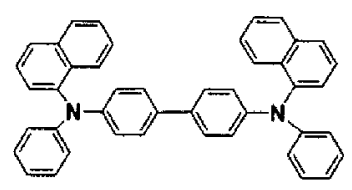

$a-N P D$

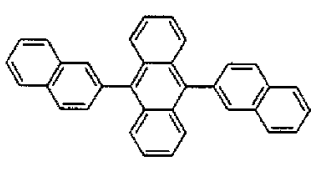

ADN

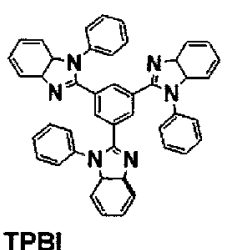

TPBI
FIG. 2. Chemical structures of materials used: E3, $\alpha$-NPD, ADN, TPBI.

tion source, a large ionization potential and a small electron affinity for hole injection and electron injection into most organic materials, respectively, and efficient bipolar carrier transport (high carrier mobilities for both holes and electrons) without trapping photogenerated carriers. These requirements indeed point to a large-gap ambipolar chargetransport material.

As such, the recent finding that large-gap oligofluorenes exhibit intriguing nondispersive ambipolar carrier-transport properties and high mobilities over $10^{-3} \mathrm{~cm}^{2} / \mathrm{V} \mathrm{s}$ for both carriers strongly suggests that they may be ideal candidates as the common CGL for the TOF measurement. ${ }^{8,9}$ In this letter, we demonstrate the use of a terfluorene in the CGLTOF technique to measure both hole and electron mobilities of various organic materials.

Figure 2 shows the chemical structures of materials used in this work. Terfluorene E3 used in this study has ethyl substitutions on the C9 carbon. E3 has a reasonably high glass transition temperature of $104^{\circ} \mathrm{C}$ and thereby forms homogeneous and stable amorphous films with vacuum deposition. The absorption spectrum of the vacuumdeposited E3 film is shown in Fig. 3, in which large absorption coefficients of $>10^{5} \mathrm{~cm}^{-1}$ are observed at $325-375 \mathrm{~nm}$. Such wavelengths match those of the frequency-tripled Nd:YAG laser $(355 \mathrm{~nm})$ and the nitrogen laser $(337 \mathrm{~nm})$, two affordable and reliable nanosecond pulsed laser systems widely used in the TOF measurements. For the frequencytripled Nd:YAG laser (355 nm, $10 \mathrm{~ns}$ pulse width) used in this study, the absorption coefficient is up to $1.3 \times 10^{5} \mathrm{~cm}^{-1}$. Figures 4(a) and 4(b) show the representative TOF transients of holes and electrons, respectively, for E3 measured at room temperature using the TOF sample configuration of glass substrate/Ag $(30 \mathrm{~nm}) / \mathrm{E} 3(1.5 \mu \mathrm{m}) / \mathrm{Al}(150 \mathrm{~nm})$. Both tran-

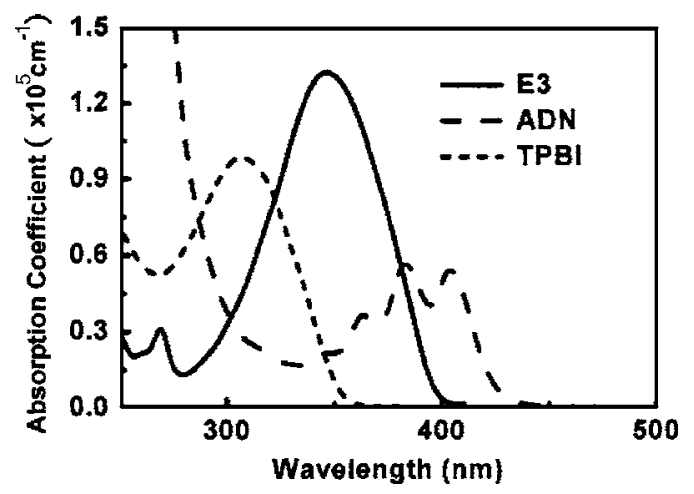

FIG. 3. Absorption spectra of vacuum-deposited E3, ADN, and TPBI films. Downloaded 17 Feb 2009 to 140.112 .113 .225 . Redistribution subject
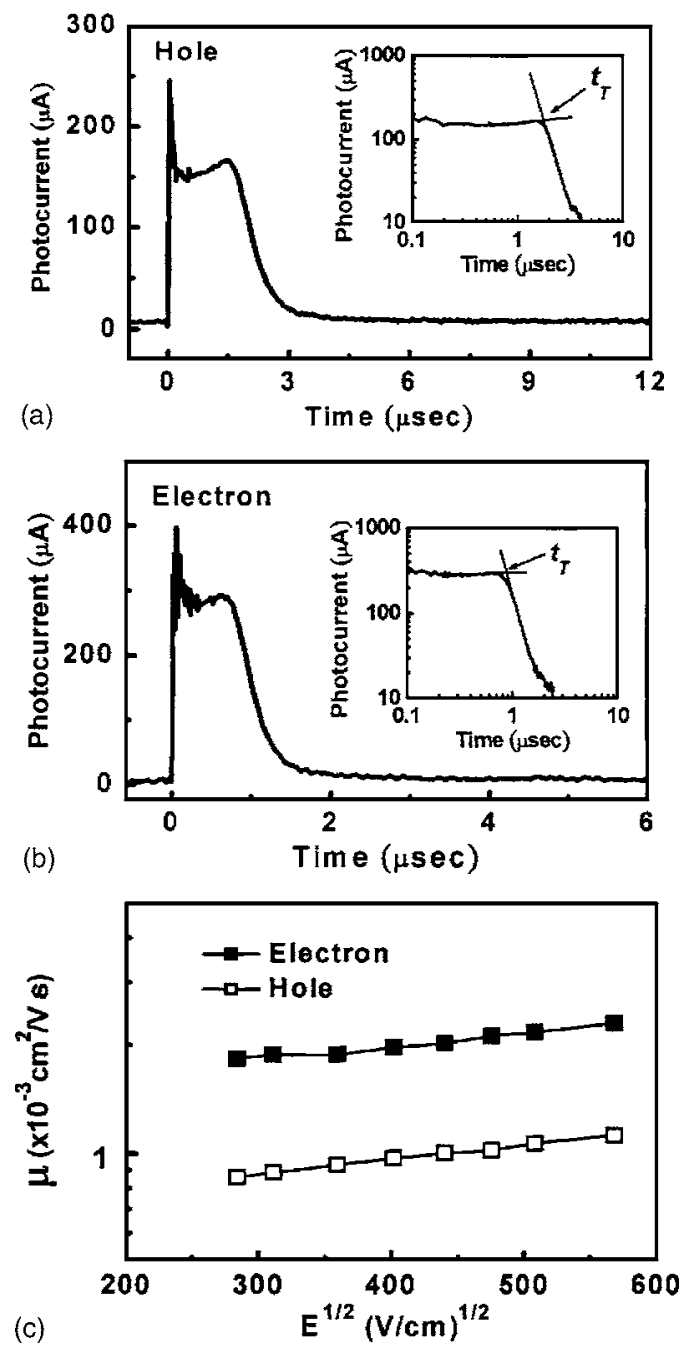

FIG. 4. Representative TOF transients for E3 $(1.5 \mu \mathrm{m})$ : (a) hole, $E$ $=10^{5} \mathrm{~V} / \mathrm{cm}$; (b) electron, $E=10^{5} \mathrm{~V} / \mathrm{cm}$. Insets of (a)-(b) are double logarithmic plots of (a)-(b), respectively. (c) Hole and electron mobilities vs $E^{1 / 2}$ for E3.

sients show a clear constant-current plateau followed by a rapid falloff, a clear signature of nondispersive carrier transport. ${ }^{7}$ Using the carrier transit time $t_{T}$ unambiguously evaluated from the intersection point of the asymptotes to the plateau and the tail sections in the double logarithmic representation [inset of Figs. 4(a) and 4(b)], hole and electron mobilities of E3 are determined and are shown as a function of the square root of the electric field $\left(E^{1 / 2}\right)$ in Fig. 4(c). Electron and hole mobilities up to $2.5 \times 10^{-3}$ and 1 $\times 10^{-3} \mathrm{~cm}^{2} / \mathrm{V} \mathrm{s}$ are observed. Furthermore, E3 possesses a relatively large ionization potential $\left(I_{p}\right)$ of $\sim 5.9 \mathrm{eV}$ in thin films, as determined by the ultraviolet photoemission spectroscopy (UPS) under high vacuum. ${ }^{10}$ With the optical energy gap of $3.2 \mathrm{eV}$ (taken from the absorption onset of E3), the electron affinity (EA) of E3 is estimated to be $2.7 \mathrm{eV}$. Such energy levels of E3 suggest that it shall be feasible for photogenerated carriers (either holes or electrons) in E3 to inject into a wide range of organic materials commonly used in organic devices.

To demonstrate the usefulness of E3 as the general CGL for the TOF measurement, three materials often used in organic light-emitting devices (OLEDs) (Fig. 2), the wellknown hole-transport material $\alpha$-naphthylphenylbiphenyl diamine $(\alpha$-NPD), the blue-emitting material 9,10-di-(2to AIP license or copyright; see http://apl.aip.org/apl/copyright.jsp 

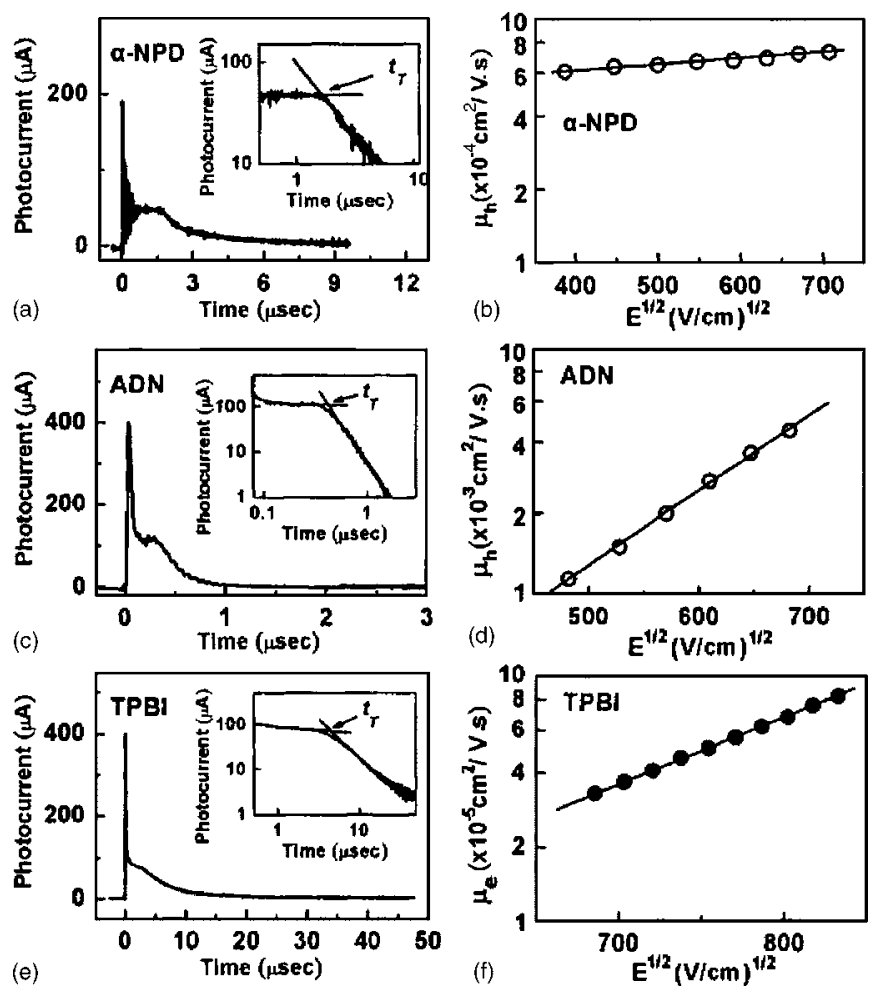

FIG. 5. (a) Representative TOF transients for holes of $\alpha$-NPD at $E=2$ $\times 10^{5} \mathrm{~V} / \mathrm{cm}$. (b) Hole mobilities vs $E^{1 / 2}$ for $\alpha$-NPD. (c) Representative TOF transients for holes of ADN at $E=3.3 \times 10^{5} \mathrm{~V} / \mathrm{cm}$. (d) Hole mobilities vs $E^{1 / 2}$ for ADN. (e) Representative TOF transients for electrons of TPBI at $E=7 \times 10^{5} \mathrm{~V} / \mathrm{cm}$. (f) Electron mobility vs $E^{1 / 2}$ for TPBI. Insets of (a), (c), and (e) are double logarithmic plots of (a), (c), and (e), respectively.

naphthyl)anthracene (ADN) and the electron-transporting material 1,3,5-tri(phenyl-2-benzimidazoly)-benzene (TPBI) ${ }^{11-14}$ had been tested using the sample structure of glass substrate/Ag $(30 \mathrm{~nm}) / \mathrm{E} 3(0.2 \mu \mathrm{m}) / \alpha$-NPD, ADN or TPBI $(2 \mu \mathrm{m}) / \mathrm{Al}(150 \mathrm{~nm})$. It is interesting to note that although ADN and TPBI are widely used in OLEDs, there are no reports of direct characterization of their carrier-transport properties with the TOF technique. As shown in Fig. 3, the excitation wavelength of $355 \mathrm{~nm}$ is close to the absorption onset of TPBI and the absorption minimum between absorption bands of ADN. Hence at $355 \mathrm{~nm}$, both TPBI and ADN have rather small absorption coefficients of $\sim 5 \times 10^{3}$ and $\sim 2.4 \times 10^{4} \mathrm{~cm}^{-1}$, respectively. Without using a CGL, it would require an impractical sample thickness of $>10 \mu \mathrm{m}$ to obtain accurate TOF results for these materials. In contrast, by incorporating E3 as the CGL, a more practical thickness of $2 \mu \mathrm{m}$ for ADN and TPBI was used in the TOF sample configuration. The thicknesses of organic materials under testing were still set substantially larger than that of E3 so that the total transit time is larger than the time resolution of the electronic system and the transit time across the E3 layer is negligible in comparison with the total transit time.

Figure 5(a) shows the representative TOF transient for holes of $\alpha$-NPD, in which the constant-current plateau indicates its typical nondispersive hole transport. Using the transit time $t_{T}$ determined from the TOF transients [inset of Fig. $5(\mathrm{a})$ ], hole mobilities of $\alpha$-NPD are determined and are shown as a function of $E^{1 / 2}$ in Fig. 5(b). The mobilities measured with the current CGL-TOF method are consistent with previously reported values, ${ }^{12}$ indicating the reliability of the current approach. Figure 5(c) shows the representative TOF transient for holes of ADN, which exhibits nondispersive hole-transport characteristics. On the other hand, the TOF transient for electrons of ADN is highly dispersive and no transit time and electron mobility could be extracted. As shown in Fig. 5(d), ADN exhibits a high hole mobility of $1-4.5 \times 10^{-3} \mathrm{~cm}^{2} / \mathrm{V} \mathrm{s}$ at $E=2.3-4.7 \times 10^{5} \mathrm{~V} / \mathrm{cm}$, which is indeed higher than that of $\alpha$-NPD. In the common blue OLEDs based on sandwiching the blue-emitting ADN between the hole-transport arylamine and the electron-transport tris(8-hydroxyquinoline)aluminum $\left(\mathrm{Alq}_{3}\right)$, degradation of color purity of blue emission due to partial green emission from $\mathrm{Alq}_{3}$ is often observed. ${ }^{11,14}$ Such phenomena may be more understandable with the high hole mobility of ADN reported here, since some holes injected into ADN may transport throughout the whole layer and recombine with electrons in $\mathrm{Alq}_{3}$.

On the other hand, slightly dispersive electron transport was observed for TPBI [Fig. 5(e)], yet no hole transport was observed. As shown in Fig. 5(f), TPBI has an electron mobility of $3.3-8 \times 10^{-5} \mathrm{~cm}^{2} / \mathrm{V} \mathrm{s}$ at $E=4.7-7 \times 10^{5} \mathrm{~V} / \mathrm{cm}$, which is consistent with those measured by transient electroluminescence and is about one order of magnitude higher than that of the widely used electron-transport material $\mathrm{Alq}_{3} \cdot{ }^{14,15}$ It is consistent with previous observations that replacing $\mathrm{Alq}_{3}$ with TPBI as the electron-transport layer in OLEDs usually reduces the operation voltage. . $^{13,14}$

In summary, we have shown that oligofluorenes are useful as the general charge-generation materials for the TOF measurement of both hole and electron mobilities of organic materials. Such a scheme shall have the advantages of simplifying the instrumentation and reducing material consumption in the TOF mobility measurements.

The authors would like to acknowledge financial support from National Science Council of Republic of China.

${ }^{1}$ C. W. Tang and S. A. VanSlyke, Appl. Phys. Lett. 51, 913 (1987).

${ }^{2}$ C. W. Tang, Appl. Phys. Lett. 48, 183 (1986).

${ }^{3}$ J. H. Burroughes, D. D. C. Bradley, A. R. Brown, R. N. Marks, R. H. Friend, P. L. Burns, and A. B. Holmes, Nature (London) 347, 539 (1990).

${ }^{4}$ A. A. Shoustikov, Y. You, and M. E. Thompson, IEEE J. Sel. Top. Quantum Electron. 4, 3 (1998).

${ }^{5}$ F. Garnier, G. Horowitz, X. Peng, and D. Fichou, Adv. Mater. (Weinheim, Ger.) 2, 592 (1990).

${ }^{6}$ T. N. Jackson, Y.-Y. Lin, D. J. Gundlach, and H. Klauk, IEEE J. Sel. Top. Quantum Electron. 4, 100 (1998).

${ }^{7}$ P. M. Borsenberger and D. S. Weiss, Organic Photoreceptors for Imaging Systems (Marcel Dekker, New York, 1993).

${ }^{8}$ C.-C. Wu, T.-L. Liu, W.-Y. Hung, Y.-T. Lin, K.-T. Wong, R.-T. Chen, Y.-M. Chen, and Y.-Y. Chien, J. Am. Chem. Soc. 125, 3710 (2003).

${ }^{9}$ C.-C. Wu, T.-L. Liu, Y.-T. Lin, W.-Y. Hung, T.-H. Ke, K.-T. Wong, and T.-C. Chao, Appl. Phys. Lett. 85, 1172 (2004).

${ }^{10}$ C. I. Wu, Y. Hirose, H. Sirringhaus, and A. Kahn, Chem. Phys. Lett. 272, 43 (1997).

${ }^{11}$ J. Shi and C. W. Tang, Appl. Phys. Lett. 80, 3201 (2002).

${ }^{12}$ S. Naka, H. Okada, H. Onnagawa, Y. Yamaguchi, and T. Tsutsui, Synth. Met. 111-112, 331 (2000).

${ }^{13}$ Y. T. Tao, E. Balasubramaniam, A. Danel, and P. Tomasik, Appl. Phys. Lett. 77, 933 (2000).

${ }^{14}$ Y. Q. Li, M. K. Fung, Z. Y. Xie, S. T. Lee, L. S. Hung, and J. M. Shi, Adv. Mater. (Weinheim, Ger.) 14, 1317 (2002).

${ }^{15}$ G. G. Malliaras, Y. Shen, D. H. Dunlap, H. Murata, and Z. H. Kafafi, Appl. Phys. Lett. 79, 2582 (2001). 